\title{
Les fondements socioculturels de l'Etat moderne
}

\section{Thierry Michalon}

\section{OpenEdition \\ Journals}

Édition électronique

URL : http://journals.openedition.org/plc/327

DOI : $10.4000 /$ plc.327

ISSN : 2117-5209

\section{Éditeur}

L'Harmattan

Édition imprimée

Date de publication : 1 janvier 2000

Pagination : 59-77

ISSN : 1279-8657

\section{Référence électronique}

Thierry Michalon, «Les fondements socioculturels de l'Etat moderne », Pouvoirs dans la Caraïbe [En ligne], 12 | 2000, mis en ligne le 14 mars 2011, consulté le 19 avril 2019. URL : http:// journals.openedition.org/plc/327 ; DOI : 10.4000/plc.327 


\title{
Les fondements socio-culturels de l'Etat moderne
}

\author{
Par Thierry MICHALON \\ Maître de conférences en droit public \\ Université des Antilles et de la Guyane \\ Faculté de Droit et d'Economie de \\ Martinique
}

Si l'Etat constitue le cadre de notre vie quotidienne, en fournir une définition satisfaisante n'est pas une mince affaire. En témoigne, par exemple, le désarroi des commentateurs devant les atteintes multiformes à l'ordre public dont la Corse est le théâtre depuis un quart de siècle. "L'Etat est-il présent en Corse ? » fut une manchette fréquente, traduisant l'angoisse qu'engendre une situation où, de toute évidence, les institutions de la République, quoique sur-représentées eu égard à la faiblesse de la population - s'avèrent incapables d'obtenir le respect de la loi. Un tel divorce entre la présence physique des institutions et la faible effectivité de la règle de droit qu'elles sont chargées de mettre en œuvre vient en effet ruiner la vision essentiellement institutionnelle de l'Etat dont nous sommes porteurs, et nous contraint à une réflexion plus délicate car plus perturbante, en termes de légitimité.

Les définitions traditionnelles de l'Etat, en effet, sempiternellement reproduites par des auteurs soucieux de s'inscrire dans des traditions ressenties comme prestigieuses, s'avèrent aujourd'hui de peu de secours pour expliquer tant l'échec des tentatives faites pour greffer l'Etat moderne dans les anciens territoires coloniaux que la relative réussite de cette greffe dans les vieilles sociétés industrialisées. La plus ressassée de ces définitions, héritée de Carré de Malberg, présente, on le sait, l'Etat comme la réunion des trois éléments que sont: un territoire délimité, une population 
déterminée, une organisation politique souveraine ${ }^{1}$. Ces trois notions résistent aujourd'hui fort mal à l'analyse ${ }^{2}$.

Une définition moins fréquente franchit mieux l'examen, mais conduit à des pratiques politiques également incapables d'assurer l'effectivité de l'Etat de droit : l'Etat, personnification juridique de la nation. L'Etat est ici désigné comme la première personne morale, celle qui donne vie à la nation sur le plan du droit, en lui permettant de se doter d'institutions exprimant sa volonté, d'organiser la vie collective par l'édiction de règles, d'établir des rapports formalisés avec les autres nations érigées en Etats. La notion de nation, présentée par Renan, on le sait, comme un vouloir-vivre collectif, comme un sentiment partagé, constitue le talon d'Achille de cette définition, qui entraîna les jeunes Etats issus du processus de décolonisation dans de graves impasses. Elle incite en effet à chercher le fondement de l'Etat dans une solidarité, dans un consensus national, que les dirigeants s'efforcèrent de forger de manière accélérée - s'agissant de peuples réunis par une brutale et brève histoire coloniale - au moyen des techniques de la démocratie unanimitaire. Election du chef de l'Etat au suffrage universel (les prestigieux précédents américain et français étant ici pain bénit pour les candidats-dictateurs), parti unique ou tout au moins dominant, intimidation-séduction de l'élite, achat des opposants par des emplois administratifs voire ministériels, usage constant d'une rhétorique mobilisatrice culpabilisant par avance toute réticence, tous les procédés ont été mis en œuvre pour tenter d'accoucher d'un sentiment national aux forceps, et de fournir ainsi à l'Etat le substrat politique, le consensus, sur lequel on pensait qu'il devait trouver son assise.

L'échec de ces procédés est aujourd'hui avéré, qu'il s'agisse des Etats issus de la décolonisation ${ }^{3}$, dont beaucoup sont en pleine

1. Cf. notamment Mekhantar J., Droit politique et constitutionnel, éd. ESKA, 1997 , p. 40 et s.

2. Voir la contribution à ce dossier de Bernard Vonglis : «L'Etat, définitions et réalités ».

3. Certains, dont l'auteur de ces lignes, ont alors proposé, s'agissant des Etats africains, de rompre avec l'attitude ultra-jacobine consistant à attendre de l'Etat unitaire qu'il accouche d'une solidarité nationale, au profit d'une approche fédéraliste 
désagrégation (Afrique sub-saharienne, Algérie dans une certaine mesure) qu'il s'agisse aussi des Etats «socialistes »: l'unanimité de commande n'a pu conférer aux institutions la légitimité sans laquelle il est vain d'escompter des hommes le respect des règles qu'elles émettent.

De la même manière, proclamer l'appartenance à la Nation des «vieilles colonies » et de la Corse, et croire l'avoir définitivement concrétisée par le statut départemental, a conduit l'Hexagone à des désillusions, dont il ne prend acte que dans la douleur tant la vision positiviste de notre droit demeure fermée à toute réflexion en termes de légitimité.

Il importe donc de partir de nouveau à la recherche de la nature de l'Etat moderne, ou plus exactement de ses fondements. On propose ici de les rechercher dans une analyse des fonctions qu'il remplit au sein du corps social. Ces fonctions se ramènent, peut-on penser, à une fonction politique, consistant à enregistrer les attentes majoritaires s'exprimant dans celui-ci pour les transformer, par le truchement des procédures démocratiques, en règles de vie collective (le droit), et à une fonction administrative, consistant à mettre ces règles en application en traitant de manière identique les administrés se trouvant dans des conditions identiques, comme l'exigent les valeurs républicaines.

tendant au contraire à construire les institutions autour du schéma des solidarités existantes, de nature communautaire, régionale. Cf. Michalon Th., Quel Etat pour l'Afrique ?, Ed. L'Harmattan, 1984. Ces propositions de fédéralisme ethnique se sont heurtées aux vives réticences des élites africaines, peu soucieuses de quitter les capitales actuelles - où chacun peut établir envers sa communauté d'origine le minimum de distance permettant un peu de cette affirmation de soi dont la civilisation occidentale donne l'exemple envié - pour retourner dans des capitales régionales, ethniquement homogènes, où chacun serait absorbé de nouveau par le puissant contrôle social des siens. La voie fédérale est donc d'emblée disqualifiée par la régression dont elle s'accompagnerait aux yeux d'hommes et de femmes ayant goûté, du fait de leur formation, à l'autonomie de la personne. 


\section{I. - LA GESTION PACIFIQUE DES CONFLITS D'INTERETS, FONDEMENT DE LA PROCEDURE DEMOCRATIQUE}

Renvoyant par étymologie au pouvoir $d u$ peuple, au gouvernement de la Cité par l'assemblée des citoyens sur l'agora, par opposition - selon Hérodote déjà ${ }^{5}$ - à la monarchie et à l'aristocratie, la notion de démocratie évoque un mode d'exercice enfin pacifié du pouvoir, dépouillé de l'arbitraire d'un prince comme de la domination d'une élite. Telle est bien d'ailleurs, aujourd'hui, l'image que se font des démocraties occidentales les peuples qui, tels les peuples d'Afrique, peinent à se libérer de leurs traditionnels affrontements communautaires : à leurs yeux, la démocratie a pu éclore dans les sociétés du « Nord » car ces sociétés seraient relativement homogènes, donc peu conflictuelles, et qu'il règnerait en leur sein un consensus fondamental. $\mathrm{Au}$ sein du monde occidental lui-même, les cultures latines, peu enclines à sacrifier la liberté de la personne aux disciplines collectives, apparaissent aux cultures anglo-saxonnes ou scandinaves, où le contrôle social occupe une plus grande place, comme laissant trop d'espace aux antagonismes pour pouvoir se réclamer de la démocratie moderne ${ }^{6}$. L'idée de démocratie semble donc bien liée à une image de paix et de consensus.

Très répandu, ce sentiment est trompeur et égare nombre d'élites vers les tentations du rassemblement voire de l'unanimité.

Or la démocratie n'est pas le consensus. Elle suppose au contraire l'antagonisme des attentes. Elle est la procédure permettant d'élaborer pacifiquement un compromis entre ces attentes et de traduire ce compromis en règles de vie collective nouvelles. Et les sociétés caractérisées non par l'antagonisme des attentes mais par les

4. «Lorsque, dans la république, le peuple en corps a la souveraine puissance, c'est une démocratie. », Montesquieu, De l'Esprit des Lois, Livre II, chap. II.

5. Raynal J.-J., « Histoire des grands courants de la pensée politique », Hachette, Les Fondamentaux, 1999, p. 9.

6. Qui n'a encore en mémoire le mépris avec lequel les autorités monétaires allemandes stigmatisaient naguère le laxisme caractérisant à leurs yeux la gestion des « pays du Club Med. »... 
affrontements communautaires ne peuvent que détourner les procédures démocratiques.

\section{A. - La démocratie n'est pas le consensus}

Contrairement au sentiment répandu parmi les peuples de longue date accoutumés à la démocratie libérale, celle-ci ne va pas de soi, et ne semble pas dans la nature des choses. Seul un effort sur eux-mêmes, ressortissant à la culture, peut amener les hommes à partager la tâche complexe, perturbante, et moins gratifiante que génératrice de tensions et de frustrations, consistant à gouverner la Cité. Une sorte de fuite naturelle des conflits autant que des responsabilités nous conduirait plutôt à nous rassembler sous l'autorité réconfortante d'un chef...

Profondément naturel, en effet, l'instinct de compétition conduit à reconnaître la domination des forts, alors même qu'un désir de paix, de quiétude, comme une lâcheté foncière, nous pousse à chercher à nous en remettre à plus fort que nous, à un chef donc, chargé de trancher au lieu et place des intéressés. Cette propension n'est nullement propre aux sociétés que nous regardons comme archaïques : la grande popularité, en France, de la désignation du Président de la République au suffrage universel traduit clairement ce profond désir de chef, ce besoin de confier notre sort collectif à un homme présumé plus fort que les autres et que nous pourrons, commodément déchargés de la responsabilité de nous-mêmes, admirer ou haïr librement.

Il s'agit en même temps d'un désir fondamental de fuir les conflits, de se rassembler sous la houlette bienveillante d'un homme bon, investi, lui, de l'impossible mission d'élaborer les compromis satisfaisant tout le monde. Désir de chef, donc, mais aussi désir de consensus, car avidité de paix, comme le traduisent à l'envi les propos de campagne. Les candidats appellent au «rassemblement» des électeurs sur leur nom, promettent d'être «le Président de tous les Français », parfois même s'engagent à faire prendre des mesures satisfaisant des intérêts collectifs antagonistes. Ce faisant, ils sollicitent dans l'opinion ce rêve inavoué de consensus, d'union, d'apaisement des conflits collectifs, et entretiennent l'idée qu'un peuple mûr «sait 
faire taire ses divisions ». Et l'image consensuelle que nous recevons des peuples européens plus septentrionaux - caractérisés à la fois par un contrôle social plus fort et par une plus grande efficacité économique - ne contribue guère à nous réconcilier avec notre propre mode de gestion collectif, traditionnellement plus conflictuel.

Si les peuples inventeurs de la démocratie libérale aspirent ainsi à la fuir au profit du consensus, nulle surprise à ce que la greffe démocratique ait été jusqu'ici rejetée par les sociétés nonindustrialisées, par les cultures rurales, où l'entraide communautaire occulte l'antagonisme objectif des intérêts. A l'intérieur des clans familiaux, des communautés ethniques, les obligations ancestrales d'assistance mutuelle (les dons et contre-dons des anthropologues) font obstacle à la prise de conscience par chacun de ses intérêts propres, et de leur éventuel antogonisme avec ceux du cousin. Aussi la démocratie unanimitaire inspirée des régimes marxistes a-t-elle trouvé très vite, dans les territoires décolonisés il y a une quarantaine d'années, un terreau favorable à son épanouissement: la contrainte d'un régime autoritaire devait y être légitimée par l'efficacité prêtée au consensus, au rassemblement autour d'un programme de développement, d'un parti, d'un leader. On le sait, cette démocratie par les buts (construire une société démocratique) s'avéra incapable de susciter en faveur des institutions publiques la légitimité dont la démocratie par les moyens (le libre affrontement des intérêts en conflit) s'est au contraire montrée porteuse, et ces expériences échouèrent précisément par la faible adhésion aux institutions. Il est donc clair aujourd'hui que rien n'est possible sans l'assentiment de la majorité au niveau des méthodes d'élaboration du compromis social puis de la règle de droit. Et il est non moins clair que le consensus, nécessairement obtenu par diverses pressions même s'il semble correspondre en nous à un profond désir de quiétude et de déresponsabilisation, n'est pas la démocratie, et ne peut constituer un fondement durable de l'Etat moderne. 


\section{B. - La démocratie suppose l'antagonisme des attentes}

La démocratie procède d'un effort collectif - d'une culture, donc - allant à l'encontre d'une propension naturelle à nous en remettre à un chef... et à maugréer ensuite in pectore contre son autorité. Elle suppose la renonciation au mythe confortable de l'unité du groupe, de son homogénéité fraternelle, au profit d'une reconnaissance douloureuse des conflits collectifs qui le traversent, et l'organisation réaliste de leur libre expression. Inventée par et pour des sociétés où les solidarités verticales fondées sur l'entraide clanique cédaient rapidement la place à des solidarités horizontales fondées sur la défense d'intérêts collectifs antagonistes, la démocratie n'est pas la quiétude. Elle est l'acceptation, l'officialisation des conflits d'intérêts suscités par deux exigences objectivement contradictoires présentes dans nos sociétés, et qu'Alain Touraine résume magistralement en ces termes : «la (nécessaire) concentration des investissements et la répartition des produits de la croissance $»^{7}$, avant d'expliquer qu'elle n'est pas un état, mais une lutte, l'expression libre, par le truchement des mécanismes électifs, des revendications antagonistes des acteurs sociaux.

Très concrètement, le progrès économique suppose que certains puissent investir dans le développement des moyens de production; investir exige d'avoir épargné ; la formation d'une épargne suppose une position privilégiée dans l'allocation des revenus, donc des inégalités dans cette allocation. Dès lors l'attente première des investisseurs et des chefs d' entreprise est la liberté dans la gestion de leurs affaires, cette liberté leur permettant de maximiser les profits qu'ils espèrent tirer de leurs investissements. Simultanément toutefois, la civilisation européenne repose sur le postulat de l'égale valeur des hommes, indépendamment de leurs accomplissements ; les inégalités trop marquées y sont ressenties par les couches défavorisées comme illégitimes, et leurs membres attendent de la collectivité qu'elle y apporte un correctif en organisant elle-même, dans une certaine mesure, la répartition des richesses.

7. Touraine A., Qu'est-ce que la démocratie ?, Fayard, Paris, 1994, p. 171. 
Or liberté et égalité se sont avérées, on le sait, incompatibles comme l'eau et le feu, la liberté suscitant l'accroissement des inégalités, les mesures favorables à une plus grande égalité passant par des restrictions de libertés, au moins économiques. Et nulle part n'existe de règle objective, scientifique, permettant d'établir un compromis majoritairement satisfaisant entre ces attentes contradictoires : établir pacifiquement ce compromis, pour une société donnée, à une époque donnée, l'adapter constamment à l'évolution des rapports de force entre la demande de liberté de ceux qui se sentent forts, d'une part, et la demande d'égalité de ceux qui se sentent faibles, d'autre part, telle est précisément la raison d'être des mécanismes démocratiques, leur essence même. La démocratie libérale est donc par nature le produit d'une société stratifiée en classes sociales aux intérêts antagonistes exprimant des attentes objectivement contradictoires. Elle ne s'alimente pas de la simple rivalité de groupes sociaux rivaux certes, mais ayant des intérêts identiques : conquérir, au profit de leurs membres, la plus large partie des privilèges afférents au pouvoir, par exemple.

\section{C. - La procédure démocratique, première source de légitimité de la loi}

En régime parlementaire, l'«élection reine »- pour reprendre la désormais célèbre formule d'E. BALLADUR - demeure celle de la chambre basse du Parlement, nonobstant le recours éventuel au suffrage universel pour désigner le Chef de l'Etat. C'est qu'il s'agit bien de permettre là aux citoyens d'exprimer dans toutes leurs nuances les attentes qui sont les leurs en désignant leurs représentants au sein du pouvoir législatif, dans l'espoir d'orienter ainsi dans un sens conforme à leurs intérêts le contenu des normes à venir. S'affrontent ainsi très librement - du moins dans un scrutin proportionnel, ou au premier tour d'un scrutin majoritaire à deux tours - toutes les attentes présentes dans le corps social, dérivant elles-mêmes assez directement de la diversité des insertions socio-professionnelles. La loi naît donc non pas d'un consensus, contrairement à la représentation unanimiste dont beaucoup rêvent ou entretiennent le mythe, mais d'un compromis, 
supposant l'expression des conflits et non leur refoulement, supposant aussi qu'une minorité parfois importante du corps électoral accepte que la loi adoptée aille à l'encontre de ses attentes.

La transparence des procédés d'élaboration de ce compromis et de sa transformation en règles de droit investit ces dernières de l'indispensable légitimité sur laquelle repose l'effectivité de ces règles, donc la réalité de l'Etat de droit. Le respect quotidien de la plus grande partie des règles par la plus grande partie des administrés ne s'explique nullement, en effet, contrairement à l'opinion la plus répandue, par la crainte de la sanction, donc par la coercition. L'exemple de la Corse entre autres - le démontre avec limpidité : les institutions de la République s'avèrent depuis des décennies incapables d'y assurer le respect des lois, non point parce qu'elles y seraient confiées à des agents particulièrement incapables ou pusillanimes - thèse mise en avant par l'opinion et les élus insulaires - mais parce que ceux-ci s'y heurtent d'emblée à une insurmontable et multiforme réticence collective, celle d'un peuple de résistants ayant toujours refusé de reconnaître toute légitimité aux puissances venues de la mer, implantées sur les côtes de l'île et prétendant faire respecter leur ordre à l'intérieur de celle-ci.

Dès lors il est clair que si l'Etat se caractérise, comme l'explique Max Weber, par le monopole de la contrainte légitime, l'absence de légitimité ruine toute possibilité réelle d'exercer véritablement et durablement cette contrainte, donc prive l'Etat de toute assise durable. Et l'effondrement, de l'intérieur, des Etats socialistes à la fin des années 80, prit sa source dans la lucidité et le courage de M. Gorbatchev, renonçant à prolonger par la contrainte un système dont il reconnaissait la faible légitimité aux yeux des citoyens soviétiques $^{8}$, qu'il n'était plus possible de couper du monde extérieur et de maintenir plus longtemps dans le système d'intimidationséduction sur lequel reposent tous les régimes autoritaires.

8. «La société étouffait dans le carcan du système de commandement administratif, condamné à servir l'idéologie et à porter le terrible fardeau de la militarisation à outrance. (...) Il n'était plus possible de vivre ainsi. » Gorbatchev M., allocution télévisée du 25 décembre 1991, Le Monde, 27 décembre 1991, p. 3. 
Si le respect de la règle s'ancre bien sur sa légitimité, il importe toutefois de distinguer clairement celle-ci d'un consensus sur son contenu. C'est le propre des régimes autoritaires que s'efforcer de bâtir un unanimisme de commande autour des règles qu'ils émettent. En régime démocratique, le consensus ne porte que sur la procédure d'élaboration des règles, aucunement sur leur contenu; mais le consensus dont jouissent les institutions (démocratiques) confère une première forme de légitimité aux règles que celles-ci édictent, et expliquent en partie leur respect, leur effectivité.

\section{D. - Fondées sur des antagonismes inter-communautaires, les sociétés non-industrialisées détournent les procédures démocratiques}

La notion d'institution, tout d'abord, issue d'un effort collectif et de longue haleine pour fonder l'organisation de la Cité sur la raison et non pas sur le bon vouloir du plus fort, trouve difficilement sa place dans les sociétés restées à l'écart de la révolution industrielle et du puissant mouvement d'urbanisation qui l'accompagna, où solidarités et antagonismes ont de tous temps été fondés sur l'affectivité, regroupant les personnes apparentées - ou s'imaginant telles - dans la sphère intérieure, celle des obligations d'entraide, de dons et contre-dons, caractéristiques des cultures rurales, rejetant les autres dans la sphère extérieure, celle de la méfiance, de la rivalité, de la jalousie. Face à la précarité de leurs conditions d'existence, les sociétés rurales ont en effet de très longue date développé des systèmes de sécurité centrés sur les obligations mutuelles qu'entraînent les liens de la parenté, ressentis comme l'unique fondement possible de la confiance. Repliées sur la communauté familiale, ces sociétés n'acceptaient - et tendent toujours à n'accepter - une autorité commune, supérieure, que du bout des lèvres, recherchant confusément dans des conflits intercommunautaires constamment renouvelés, réamorcés, le ciment assurant la cohérence de leurs communautés constitutives. Elles opposent une véritable résistance passive à l'Etat, ressenti comme extérieur aux réseaux relationnels, comme trop impersonnel. Allergiques aux institutions publiques venues du Nord, ces cultures s'avèrent en même temps 
allergiques à la citoyenneté - supposant l'exercice anonyme de droits et d'obligations identiques pour tous - comme à la justice institutionnelle de l'Etat, dépourvue de légitimité car extérieure aux mécanismes communautaires de règlement des conflits.

Ce refus des institutions s'assortit d'un détournement des mécanismes démocratiques. N'ayant jamais été bouleversées par la révolution industrielle et l'urbanisation massive qu'elle entraîna, les cultures demeurées rurales ont conservé des solidarités de type vertical - les communautés d'esssence familiale regroupant des personnes de niveaux socio-professionnels parfois très différents unies par des obligations d'échanges mutuels de services - et ne basculent que lentement vers le schéma horizontal des classes sociales unies par des intérêts socio-économiques partagés. Il n'y aurait rien là de déplorable si les mécanismes de la démocratie libérale n'avaient été, précisément, conçus par et pour des sociétés marquées par l'antagonisme des intérêts et des attentes des différentes classes, ces mécanismes ayant pour but de permettre la libre expression de ces attentes pour en dégager pacifiquement, au moyen du suffrage, un compromis, lequel sera traduit en règles de droit nouvelles.

Ce qui s'affronte en réalité, sur la scène politique de maintes sociétés non-industrialisés ou récemment industrialisés, ce ne sont pas des visions différentes de l'avenir de la collectivité, mais des clans désireux d'accéder au pouvoir afin de faire bénéficier leurs membres des privilèges qui y sont attachés. Pour ces cultures les institutions publiques ne représentent pas le lieu où se prennent des décisions d'intérêt général et où se gèrent les services publics, mais un gisement de richesses sans maître (richesses matérielles ou symboliques) à se répartir au sein des réseaux d'entraide traditionnels : de leur point de vue, l'Etat (ou la collectivité décentralisée) est ainsi, en quelque sorte, quelquechose de comestible ${ }^{9} \ldots$ La condamnation réitérée et moralisatrice de la «corruption » par la Banque mondiale n'y pourra mais. Et la vie «politique » locale se trouve donc à la fois dépolitisée, car l'enjeu n'est pas le choix d'un avenir pour la Polis, et

9. Voir notamment Bayart J.-F., L'Etat en Afrique. La politique du ventre, Fayard, 1989. 
surconflictuelle, par le constant affrontement des clans avides de s'emparer des attributs du pouvoir, fascinants pour des sociétés de tout temps habituées à la rareté, dans le but de les répartir entre leurs membres ${ }^{10}$. Il s'agit bien là d'un véritable dévoiement des procédures démocratiques, conséquence non point de l'immoralité des élites locales, mais de leur vision du monde, autrement dit de leur culture. Et un tel dévoiement, naturellement, prive les règles émises par les institutions ainsi constituées d'une véritable légitimité démocratique, ces règles étant perçues comme prises exclusivement dans l'intérêt du clan au pouvoir.

\section{II. - L'EGALITE DE TRAITEMENT, FONDEMENT DES VALEURS REPUBLICAINES}

L'élaboration transparente des règles de droit par le truchement des procédures démocratiques leur confère une légitimité partielle, insuffisante à elle seule - on le constate dans certains Etats, par exemple au Maroc - à en assurer le respect. C'est qu'une seconde condition doit être remplie pour que la règle apparaisse plus complètement légitime aux yeux des administrés : son application égalitaire aux personnes se trouvant dans la même situation, par-delà l'existence éventuelle de liens personnels entre administrés et administrateurs. Or une telle neutralité administrative est inconcevable dans les sociétés traditionnellement fondées sur des échanges de services intra-communautaires.

10. On trouvera sur ce registre de passionnantes analyses du mode de fonctionnement des sociétés méditerranéennes, partiellement transposables à d'autres sociétés, dans Giudici N., Le Crépuscule des Corses, Grasset, 1997. 


\section{A. - L'égalité devant la règle, pivot des valeurs de la république}

La liberté est la plus visible des valeurs autour desquelles la philosophie des Lumières a organisé le monde nouveau, mais elle n'est peut-être pas la plus importante. Le postulat selon lequel un homme en vaut un autre, indépendamment de ses accomplissements, fut - et demeure toujours - probablement plus révolutionnaire encore, eu égard à la puissance des hiérarchies et des mécanismes d'allégeance ayant de tous temps structuré les sociétés traditionnelles. Et la Révolution française semble avoir clairement conçu que seule la destruction des solidarités spontanées, parcellaires, locales, de nature affective, fondées sur les liens communautaires, permettrait l'adhésion de chacun aux valeurs fondatrices de l'ordre nouveau, fondé sur la Raison, et établissant l'égalité aussi bien que la liberté.

Le projet de découpage administratif du territoire conçu par l'abbé Siéyès et présenté devant l'Assemblée constituante le 29 septembre 1789 par le député Thouret incarna jusqu'à la caricature cette obsession d'égalité. Il s'agissait de plaquer sur le territoire national une structure administrative uniforme prenant la forme d'une grille parfaitement régulière: quatre-vingt-un départements carrés identiques, divisés chacun en neuf cantons carrés, comptant à leur tour chacun neuf cantons carrés. Manifestation sans nuance de l'esprit cartésien et de la volonté de rationaliser l'organisation du pays, ce découpage devait, dans l'esprit de ses concepteurs, «disloquer » définitivement la mosaïque hétérogène des circonscriptions de l'Ancien Régime, briser la multiplicité des affinités locales pour détruire l' « esprit de province » et faire émerger «un véritable esprit national » (Thouret), indispensable fondement de l'ordre nouveau. Cette rigoureuse uniformité institutionnelle devait être la garante de ce qui, avant même la liberté, apparaissait comme la valeur centrale de la société nouvelle : l'égalité de tous devant la loi ${ }^{11}$.

11. Darcy G., «Unité et rationalité dans la construction révolutionnaire », in Moreau J. et Verpeaux M.(dir.), Révolution et Décentralisation, Economica, 1992, p. 47 à 80 . 
Si ce découpage ne fut pas retenu, l'esprit qui le sous-tendait anima pourtant toute l'œuvre administrative de la Révolution, puis celle des Premier et Second Empires, enfin celle de la III $^{\text {ème }}$ République : la destruction du tissu relationnel local conditionne la naissance du citoyen, de l'administrateur comme de l'administré, mais aussi de l'agent économique « libéral ».

Aujourd'hui, Alain Touraine estime que la «culture républicaine à la française » recherche avant tout l'unité de la Nation autour d'un projet rationnel écartant les traditions, les privilèges, les particularismes, au profit de l'égalité de traitement de citoyens égaux en droits ${ }^{12}$. Et, en-dehors de l'exemple français, particulièrement marqué, on le sait, par les conceptions jacobines, il apparaît assez clair que l'Etat moderne demeure une fiction lorsque la persistance de réseaux relationnels entre administrateurs et administrés vide de tout contenu la notion d'égalité devant la loi.

\section{B. - L'égalité devant la règle suppose l'estompement des liens affectifs}

La faible intensité de la vie de relation dans les cités des pays industrialisés n'est que partiellement due à des conditions matérielles d'existence défavorables aux relations personnelles: elle s'enracine dans des éléments plus fondamentaux. En premier lieu, la compétition et la concurrence constituent aujourd'hui la trame non seulement de la vie professionnelle mais aussi de l'ensemble des rapports sociaux : être jugé faible représente dès lors un risque de chaque instant, dont les conséquences, réelles ou imaginaires, peuvent être ressenties comme désastreuses. Chacun évite donc de se livrer, à l'occasion d'échanges personnels, et reste sur son quant-à-soi, maintenant délibérément avec autrui une distance protectrice. En second lieu, et en-dehors même de ce contexte général de compétition, chacun ressent comme une

12. Récente mais extrêmement éclairante, la distinction entre démocratie et république a fait l'objet d'une passionnante analyse de la part d'Alain Touraine, in Qu'est-ce que la Démocratie ?, Fayard, 1994. 
libération le fait d'échapper à l'omniprésent contrôle social caractérisant les cultures rurales et de vivre à sa guise, affranchi de la constante censure du regard d'autrui, comme des pesantes et immuables hiérarchies des sociétés communautaires.

La recherche de cette distance, condition de la neutralité dans la mise en œuvre des règles par les administrations donc de l'égalité de traitement des administrés, sous-tend dans la pratique ces innombrables précautions institutionnelles que les Etats modernes s'efforcent de mettre en œuvre, que sont notamment le secret du vote, les procédés de passation des marchés publics, le recrutement fréquent de la fonction publique par concours, l'anonymat des copies d'examen, le «dépaysement » de certaines affaires judiciaires, l'affectation des agents publics fraîchement recrutés à l'autre extrémité du territoire, les fréquents mouvements préfectoraux... C'est donc bien que cette distance, engendrant un certain anonymat, apparaît comme une nécessaire composante d'une organisation sociale fondée sur une éthique fonctionnelle et non plus relationnelle, sur la neutralité et l'égalité dans l'accomplissement par chacun de ses fonctions. Là semble résider la condition de l'égalité de traitement dans l'application des règles de droit comme dans les prestations des services publics, plutôt que dans la moralité supposée des agents publics ou le sens de l'intérêt général qui leur est souvent prêté.

\section{C. - L'égalité de traitement, seconde source de légitimité de la règle}

Si son élaboration au moyen des procédures démocratiques constitue pour la loi, on l'a vu, une première source de légitimité, son application dans des conditions conférant aux administrés une certitude raisonnable d'être traités sur un pied d'égalité représente sans nul doute une seconde source de légitimité. Proclamée par la Déclaration des Droits de l'Homme et du Citoyen, l'égalité devant la loi - comme devant l'ensemble des règles de droit - est bel et bien l'un des éléments fondamentaux du « pacte républicain » et, plus généralement, de l'Etat de droit. L'image très forte de l'institution préfectorale (« le 
préfet devra être un empereur au petit pied », proclama Napléon), dans notre pays comme dans ceux qui se sont inspirés de notre organisation administrative, lui a été conférée précisément par une formation, un mode de recrutement, un statut, et une gestion la maintenant résolument à l'écart des réseaux relationnels locaux et lui permettant ainsi une neutralité et une objectivité dans la mise en œuvre des règles - comme dans la gestion des services publics - que l'administré juge précieux et qui compensent à ses yeux l'aspect autoritaire et unilatéral d'un tel mode d'administration.

La réponse, bouleversante de lucidité, faite il y a quelques années par un petit commerçant marocain à ce voyageur qui l'interrogeait sur son pays, en dit long sur le caractère primordial de l'égalité de traitement : «Monsieur, au Maroc, nous avons tout ce qu'il nous faut. Il ne nous manque qu'une chose : la Loi ». Dans un Etat où fonctionnait un parlement librement élu dans le cadre d'un système de pluripartisme, cette critique fondamentale portait très probablement sur l'inégalité des administrés devant la règle de droit, en fonction de son réseau relationnel et des appuis dont il pouvait ou non bénéficier pour obtenir soit des passe-droits, soit la simple application de la loi.

Et l'on se prend alors à s'interroger sur l'exigence première des hommes quant à la nature du contrat social fondant la collectivité : celui-ci porte-t-il bien, comme on le proclame à l'envi en Occident, sur leur propre participation à l'édiction de la règle de droit (la démocratie), ou bien ne porterait-il pas plutôt sur l'assurance d'un traitement égalitaire face à la règle ? En d'autres termes, ce qu'on a proposé ici de nommer légitimité républicaine ne serait-il pas le premier fondement de l'Etat de droit, la légitimité démocratique n'occupant en réalité dans les attentes des hommes qu'une place seconde? L'histoire de la formation de l'Etat moderne en France tendrait à avaliser cette hypothèse. De la Révolution jusqu'à la mise en place de la III ${ }^{\text {ème }}$ République, en 1875, nos institutions furent en effet bâties autour de la préoccupation d'égalité de traitement, le souci de permettre la participation du citoyen à l'édiction des normes étant relégué au second plan, tant au niveau national qu'au niveau local (la technique de la déconcentration étant développée bien avant celle de la décentralisation). Et l'on peut donc penser, avec Alain Touraine, que 
l'Etat moderne fut d'abord républicain - même dirigé par un monarque - car fondé sur la Nation et sur la Raison et non plus sur un principe irrationnel et inégalitaire, avant de devenir démocratique - par l'organisation de la libre expression des intérêts. Et l'on peut alors accepter sa vigoureuse formule selon laquelle «la démocratie s'est formée contre l'Etat moderne $»^{13}$.

\section{D. - Fondées sur l'échange intra-communautaire de services, les sociétés non-industrialisées ignorent la neutralité administrative}

Lot traditionnel des sociétés rurales, la précarité de leurs conditions matérielles d'existence les amena de tous temps à maintenir des mécanismes collectifs d'entraide dont nul ne pouvait s'abstraire. Partage des tâches de production, répartition des fruits du travail, gestion collective des surplus : ces obligations de dons et contre-dons assurent une certaine sécurité mais constituent de contraignantes disciplines assujettissant chacun à la famille étendue. Parfois qualifiées de «segmentaires », ces sociétés se caractérisent, on l'a dit, par des solidarités verticales unissant les cousins les uns aux autres malgré les éventuelles disparités de leurs situations socio-professionnelles. Et le détournement des mécanismes démocratiques auxquelles, on l'a vu, elles se livrent, s'assortit d'un semblable détournement des mécanismes administratifs.

Fondée sur les relations et non pas sur les fonctions, ces sociétés ne peuvent en effet guère reconnaître de légitimité aux règles de portée générale et impersonnelle, issues de la volonté d'un pouvoir anonyme et prétendant s'appliquer indistinctement à des administrés anonymes. Dans un monde où l'identité de chacun lui est conférée par la place qu'il occupe dans un réseau relationnel déterminé, la règle générale et impersonnelle (la loi, le règlement) n'a aucun sens puisqu'elle ne s'insère pas dans l'entrelacs des dons et contre-dons, des services demandés et rendus à l'intérieur d'un système communautaire donné.

13. Ibidem, p. 60. 
Dès lors, la demande et l'octroi de passe-droits sont considérés comme le mode de relation normal et légitime avec l'Administration, à plus forte raison s'il s'agit de celle de collectivités décentralisées, administrées par des élus locaux auxquels peuvent vous relier mille liens relevant de l'affectivité. En d'autres termes, les réseaux relationnels qui «font» les élections (nationales ou locales) servent ensuite de canal privilégié à la fourniture de leurs prestations par les collectivités publiques.

Ancré dans la culture, ce détournement des logiques administratives importées correspond, certes, à la vision du monde en vigueur. Mais l'inégalité foncière devant la règle, qu'il engendre, ruine la crédibilité des institutions, et prive de légitimité républicaine les normes que celles-ci feignent de mettre en application sur l'ensemble du corps social.

\section{CONCLUSION}

L'Etat moderne semble donc bien trouver son fondement dans la double légitimité de ses institutions : la légitimité démocratique et la légitimité républicaine. On peut en conséquence proposer ici la définition de l'Etat que voici : un ensemble d'institutions chargées de l'élaboration pacifique d'un compromis entre les attentes antagonistes présentes dans le corps social, et de l'édiction de règles de comportement collectif (le droit) qui seront obéies puisque légitimes car issues de ce compromis (légitimité démocratique) et mises en cuvre de manière égalitaire (légitimité républicaine). Ainsi défini, l'Etat moderne serait alors le produit de l'histoire - notamment économique - de sociétés bien précises, et serait fort difficilement transposable à des sociétés n'ayant pas connu cette histoire... ce que l'actualité nationale et internationale tend d'ailleurs à confirmer. 


\section{Résumé}

Enfermés dans une vision étroitement institutionnelle et positiviste de l'Etat, les juristes français s'avèrent incapables d'expliquer l'échec de celui-ci dans les sociétés non-industrialisées. Cet échec s'explique lorsque l'on analyse les deux fondements de l'Etat moderne que sont la gestion pacifique des conflits d'intérêts (la démocratie) et l'application égalitaire de la loi (la république).

\section{Mots-clés}

Egalité - Etat - Sociétés nonindustrialisées - Démocratie - République

\begin{abstract}
This paper is inquiring on cultural obstacles to transplant of modern State into non-industrialized societies. We are not referring here at modern State as a mere organization, laying upon the social body, but as a process of enaction and enforcement of the rules, enabling them - or not to be respected : the setting up of a compromise between conflicting interests, and equal treatment under Law.

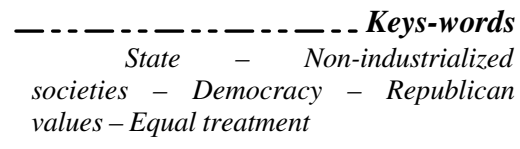

PDLC n ${ }^{\circ}$ 12, 2000 